CERN-TH.7149/94

ILL-(TH)-94-26

\title{
Phase Structure of Four Dimensional Simplicial Quantum Gravity
}

\author{
S. Catterall' \\ TH-Division, CERN CH-1211, \\ Geneva 23, Switzerland. \\ J. Kogut \\ Loomis Laboratory, University of Illinois at Urbana, \\ 1110 W. Green St, Urbana, IL 61801. \\ R. Renken \\ Department of Physics, University of Central Florida, \\ Orlando, FL 32816.
}

July 25, 2018

\begin{abstract}
We present the results of a high statistics Monte Carlo study of a model for four dimensional euclidean quantum gravity based on summing over triangulations. We show evidence for two phases; in one there is a logarithmic scaling of the mean linear extent with volume, whilst the other exhibits power law behaviour with exponent $\frac{1}{2}$. We are able to extract a finite size scaling exponent governing the growth of the susceptibility peak.
\end{abstract}

CERN-TH.7149/94

January 1994

\footnotetext{
${ }^{1}$ Permanent address: Physics Department, Syracuse University, Syracuse, NY 13244
} 


\section{Introduction}

In this article we report briefly on some new results for a model whose partition function is constructed from a sum over all random triangulations of the four dimensional sphere. Such a model has been recently studied by other groups [1, 2, 3, 4] and is a candidate for a regularised quantum theory of euclidean gravity (for fixed euler number). Specifically, the model we have looked at is defined from the partition function.

$$
Z=\sum_{T, \chi=2} e^{-\kappa_{4} N_{4}+\kappa_{0} N_{0}}
$$

The first term in the action $N_{4}$ is just the number of four simplices in the triangulation $T$ and this allows us to identify the corresponding coupling $\kappa_{4}$ as a bare cosmological constant. The second term plays the role of the integrated Ricci scalar $\left(N_{0}\right.$ is just the number of vertex labels or points in the manifold). This correspondence is clear (classically) from the usual Regge formula for the curvature in terms of the deficit angle with the extra constraint that the four simplices are all considered equilateral

$$
\int d^{4} z \sqrt{g} R=4 \pi\left(N_{0}+N_{4}-2\right)-10 \cos ^{-1}\left(\frac{1}{4}\right)
$$

Notice, also, that the formula for the local curvature at a hinge (triangle $i j k$ ) takes the form

$$
r_{i j k}=2 \pi-\cos ^{-1}\left(\frac{1}{4}\right) n_{4}^{i j k}
$$

So, if the volume is bounded the number of four simplices sharing the triangle $n_{4}^{i j k}$ is necessarily also bounded. This automatically ensures that the model is well defined at finite volume - it is a dynamical question as to whether the unboundedness problems return on taking the large volume limit.

The analogous model in two dimensions has been studied extensively, both in the continuum using analytic methods and via the triangulated lattice prescription, see for example the review [5]. It seems clear that at least for central charges less than unity, the sum over triangulated graphs correctly mimics the continuum functional integrals including the conformal anomaly. In four dimensions it is not at all clear that a simple generalisation, such as the one described above, is sufficient to explore the space of metrics. However, at this stage, it provides a convenient ansatz which may be studied using numerical simulation.

In practice, we choose to work at quasi fixed volume - that is we tune the cosmological constant $\kappa_{4}$ to fix the mean volume $\bar{N}_{4}$ to some target value $V$. To remove some of the fine tuning problems associated with this procedure we add a small correction term to the action [1] of the form

$$
\delta S=\gamma\left(N_{4}-V\right)^{2}
$$

We have verified that expectation values computed from this modified action do not depend on the coupling $\gamma$, although their errors increase as $\gamma \rightarrow 0$. In practice we have set $\gamma=0.005$ which yields an average fluctuation in the volume in the region of a fraction of a percent. 
At fixed volume, the model depends on just one parameter - the node coupling $\kappa_{0}$. As we have argued this is just inversely related to the bare Newton constant. Questions of renormalisability of continuum gravity are then replaced by the search for continuous phase transitions in this one dimensional parameter space. The hope would be that in the vicinity of such a point, it might be possible to recover a nonperturbative continuum limit for quantum gravity.

We have used a Monte Carlo algorithm to sample the triangulation space of the model. This procedure is based on a set of 'moves' or local retriangulations of the manifold, which are ergodic in the grand canonical ensemble used here. This means that it is possible to reach any triangulation from any other by sequences of these moves. In $d$ dimensions there are $d+1$ moves [1, 2] which may be pictured as the trial substitution of an $i$-subsimplex by its dual $(d-i)$-subsimplex. Our code is written in such a way as to make the dependence on dimension $d$ trivial - it enters only as an input parameter to the program [6]. This allows us to test the code in two dimensions. Finally, it is very important to ensure the update satisfies a detailed balance condition in order that the contribution of a given triangulation depends only on its Boltzmann weight.

We have simulated systems with sizes ranging from $V=500$ to $V=32000$, although the majority of our high precision data is confined to lattices of $V=8000$ and smaller. On the latter we have accumulated up to $8 \times 10^{6}$ sweeps at each value of $\kappa_{0}$ (1 sweep corresponds to $V$ attempted elementary moves).

\section{Results}

A crude order parameter which may be used to distinguish between different phases of the model is the average intrinsic linear extent of the system. This may be defined as follows

$$
L=\left\langle\frac{1}{V^{2}} \sum_{i j}^{V} d(i, j, T)\right\rangle_{T}
$$

The distance $d(i, j, T)$ on a given triangulation $T$ is just the minimal number of steps on the dual lattice between the simplex $i$ and another simplex $j$. Fig. 1 shows a plot of this for lattice volumes ranging from $V=500$ to $V=8000$. Clearly, at small $\kappa_{0}$ the typical manifolds are very collapsed and $L$ increases only very slowly with mean volume. Indeed, for fixed $\kappa_{0}$ in this range, it is possible to fit the data quite successfully $\left(\chi^{2} \sim O(1)\right)$ to a logarithm of the volume $V$.

$$
L=a\left(\kappa_{0}\right)+b\left(\kappa_{0}\right) \ln V
$$

If we care to define a fractal dimension $d_{F}$ for the system from the formula $l \sim N^{\frac{1}{d_{F}}}$, this would imply $d_{F}=\infty$. Power fits give order of magnitude worse values for the goodness-of-fit parameter $\chi^{2}$.

Conversely, at large $\kappa_{0}$ the manifolds are extended with $L$ scaling like a power of $V$ (at least for volumes up to $V=8000)$. Logarithmic fits perform much more poorly here. In fact at $\kappa_{0}=3.0$ a fit yields $d_{F}=2.08(4)$ and at $\kappa_{0}=4.0$ a value of $d_{F}=1.98(2)$. Both 
fits have $\chi^{2}$ of order the number of degrees of freedom. This fractal dimension seems to indicate that typical configurations in this phase resemble some sort of branched polymer.

We have also measured the fluctuations (per unit volume) in the mean extent $L$.

$$
\chi(L)=\frac{1}{V}\left(\left\langle L^{2}\right\rangle-\langle L\rangle^{2}\right)
$$

For small $\kappa_{0}$ this quantity is small and decreases with increasing volume. Conversely, at large $\kappa_{0}$ it plateaus at a value several orders of magnitude larger. Furthermore this asymptotic value appears to increase with volume $V$. It thus functions perhaps more effectively as an order parameter, being (for large volume) zero for a range of small couplings and very large (perhaps infinite as $V \rightarrow \infty$ ) for all large couplings. The transition between these two regions appears to become ever more sharp with increasing volume as fig. 2 indicates. It is tempting to identify the crossover point as a (pseudo) critical coupling $\kappa_{0}^{c}(V)$.

A more conventional indicator of phase transitions is the specific heat, which in this case is just the fluctuation in the number of nodes

$$
\chi\left(N_{0}\right)=\frac{1}{V}\left(\left\langle N_{0}^{2}\right\rangle-\left\langle N_{0}\right\rangle^{2}\right)
$$

This quantity is proportional to the integrated 2-pt function for the lattice scalar curvature and hence an enhancement would signal the onset of long range curvature correlations. In fig. 3 we see a sharp peak developing in the vicinity of $\kappa_{0} \sim 2$, which grows, shifts and narrows with increasing volume. Furthermore, the position of this peak coincides with the jump in the geodesic susceptibility $\chi(L)$. There is a corresponding increase in the autocorrelation time close to the peak, which we estimate grows roughly linearly with the volume. This then is our best evidence for a continuous phase transition in the system.

In systems coupled to two dimensional gravity the expectation value of some integrated quantity scales as a power of the volume, the exponent being related to the (gravitationally dressed) anomalous dimension of the matter field. Motivated by this we have looked for a

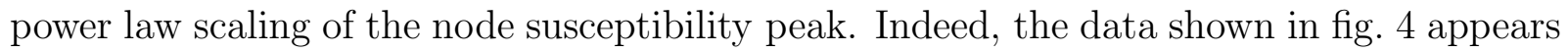
quite consistent with such a scaling scenario with a power $\Delta=0.259(7)\left(\chi^{2} \sim 1.2,29 \%\right)$. If, in addition, we assume conventional finite size scaling we might tentatively associate this power with $\Delta=\frac{\alpha}{\nu d_{F}}$ ( $\alpha$ and $\nu$ would then be the susceptibility and correlation length exponents). However the assumptions needed to establish this result may not be applicable for these systems.

Fig. 3 makes it clear that the peak in the node susceptibility is moving rather rapidly with increasing volume which opens up the (rather unpleasant!) scenario that it may diverge in the infinite volume limit. Indeed, on cursory inspection it appears possible that the pseudo critical coupling might have a component proportional to the logarithm of the mean volume $V$. The putative phase transition would then not survive the infinite volume limit and for any $\kappa_{0}$ the large volume behaviour of the model would correspond to the compact, degenerate phase. On the other hand the slow convergence could simply be the result of a small shift exponent, which might not be unreasonable given the very large intrinsic dimensionality of the compact phase.

The raw data is simply not good enough to resolve this crucial issue and so we have used multihistograming techniques $[$ to interpolate the susceptibility in the vicinity of the 
peak. The errors in the pseudo critical couplings $\kappa_{0}^{c}(V)$ were then assessed by performing several such reconstructions using simulations at different neighboring couplings $\kappa_{0}$.

To try to distinguish between these two scenarios we have attempted to fit the pseudo critical coupling $\kappa_{0}^{c}(V)$ data with both a power law form

$$
\kappa_{0}^{c}(V)=a+b V^{\omega}
$$

and a modified logarithm

$$
\kappa_{0}^{c}(V)=a+b(\ln V)^{\omega}
$$

In both cases, the converging fit $(\omega<0)$ was better and yielded statistically compatible estimates for the infinite volume critical coupling $\kappa_{0}^{c}(\infty)$. Fig. 5 shows the data, together with the result of a power fit with $a=2.9(2), b=-6.3(16)$ and exponent $\omega=-0.27(6)$. The fit yields a $\chi^{2}$ per degree of freedom of 0.2 at $85 \%$ confidence. Conventional finite size scaling would then identify the exponent $\omega=-\frac{1}{\nu d_{F}}$. By contrast the simple diverging $\log$ fit $(\omega=1)$ gave $\chi^{2} \sim O(10)$. However, one must be careful in drawing too many conclusions from this. If one drops the two smallest lattices an equally good fit can be got from the simple logarithm.

\section{Outlook}

We have presented some new results for a four dimensional model of dynamical triangulations. Emphasis has been placed on gaining some quantitative understanding of the thermodynamic properties near the putative phase transition. This has entailed accumulating two orders of magnitude more Monte Carlo data than typical earlier studies. We are able, for the first time, to convincingly resolve a finite size scaling for the specific heat peak. This exhibits a power law growth and we have been able to estimate the exponent rather accurately $\Delta=0.259(7)$. The value is inconsistent with that appropriate to a first order transition $(\Delta=1.0)$. As a further check, we have histogramed the action and have seen no evidence for the two peak structure typical of first order transitions. This evidence then favours a continuous transition.

However, as we have emphasised, the position of this specific heat peak moves rather rapidly with volume, leaving open the possibility that the pseudo critical coupling diverges with volume, the phase transition disappearing in the infinite volume limit. We have attempted to distinguish between a slow power law convergence of the critical coupling (conventional scenario) from a logarithmic or power law divergence. Since we have high statistics data with good resolution of the peak we found that histogram reconstruction was a reliable tool for the critical region and allowed us to follow the evolution of the pseudo critical couplings in more detail. The tentative conclusion from this analysis is that our data favour the conventional scenario with a finite value for the infinite volume critical coupling $\kappa_{0}^{c}(\infty)=2.9(2)$.

This transition then separates two very different phases; at small coupling $\kappa_{0}$ the entropy of small, highly connected manifolds with infinite fractal dimension dominates, at large $\kappa_{0}$, the action favours extended structures with dimension two. In this latter phase 
the fluctuations in the geodesic extent are always large and indeed yield large autocorrelation times for all $L$-dependent observables. This phase can then presumably be identified as consisting of randomly branching polymers.

It is clearly important to strengthen these conclusions by extending the analysis to larger systems. This is particularly true for the determination of the shift exponent (and the question of the finiteness of $\left.\kappa_{0}^{c}(\infty)\right)$. The present maximum volume was determined by the large autocorrelation time $O\left(10^{4}\right)$ sweeps for $V=8000$ encountered near the transition. We would hope to address this in the near future. It will then be necessary to begin to understand the relevance of the critical model to a continuum theory of gravity.

This work was supported, in part, by NSF grant PHY 92-00148. Some calculations were performed on the Florida State University Cray YMP. 


\section{References}

[1] M. Agishtein and A. Migdal, Nucl. Phys. B385 (1992) 395.

[2] J. Ambjørn and J. Jurkiewicz, Phys. Lett. B278 (1992) 42.

[3] S. Varsted, UCSD/PTH 92/03.

[4] B. Brugmann and E. Marinari, Phys. Rev. Lett 70 (1993) 1908.

[5] F. David, 'Simplicial Quantum Gravity and Random Surfaces', Saclay preprint T93/028.

[6] S. Catterall, J. Kogut and R. Renken, in preparation.

[7] A. Ferrenberg and R. Swendson, Phys. Rev. Lett 63 (1989) 1195. 


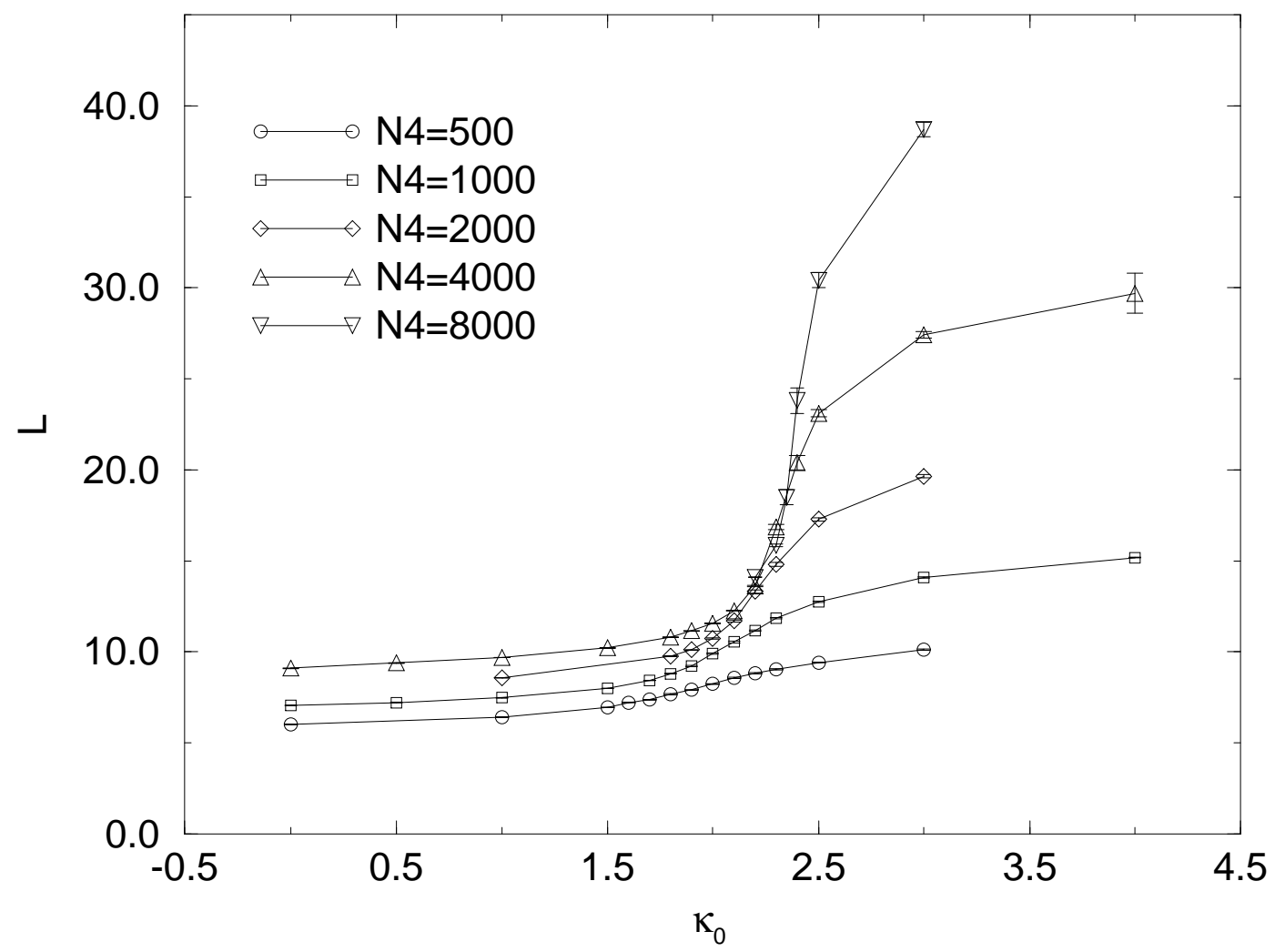

Figure 1: Mean extent 


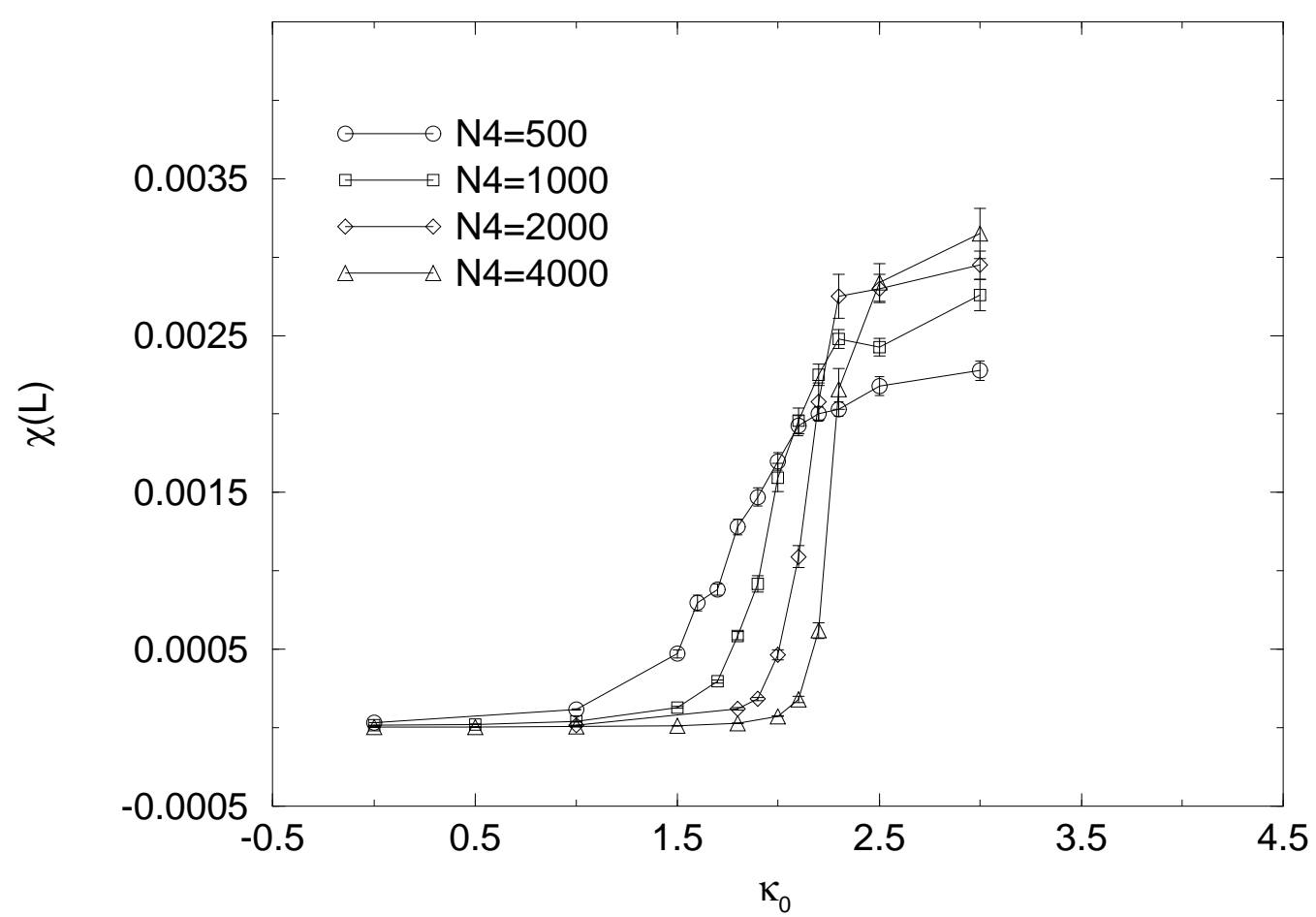

Figure 2: Fluctuation in mean extent 


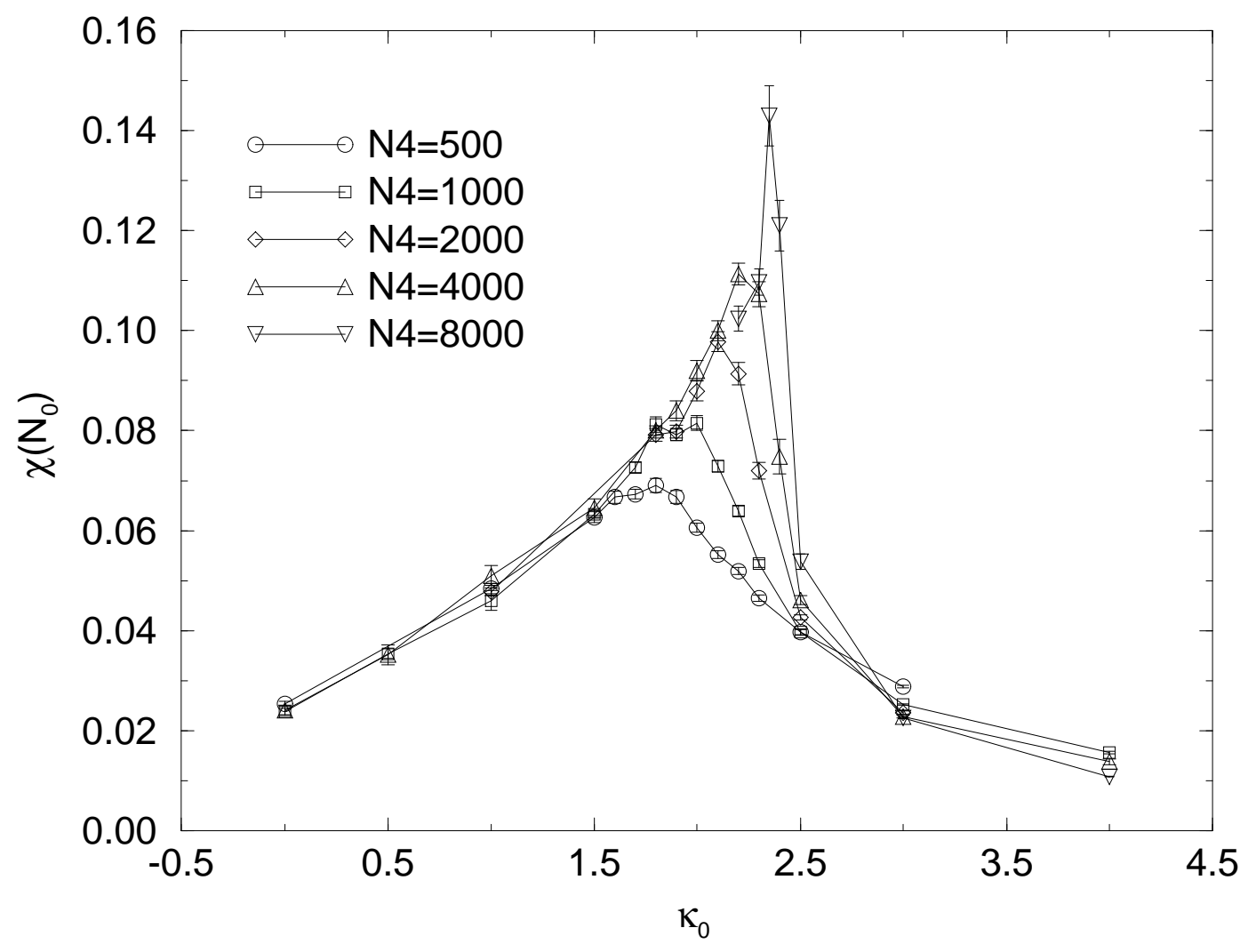

Figure 3: Node susceptibility 


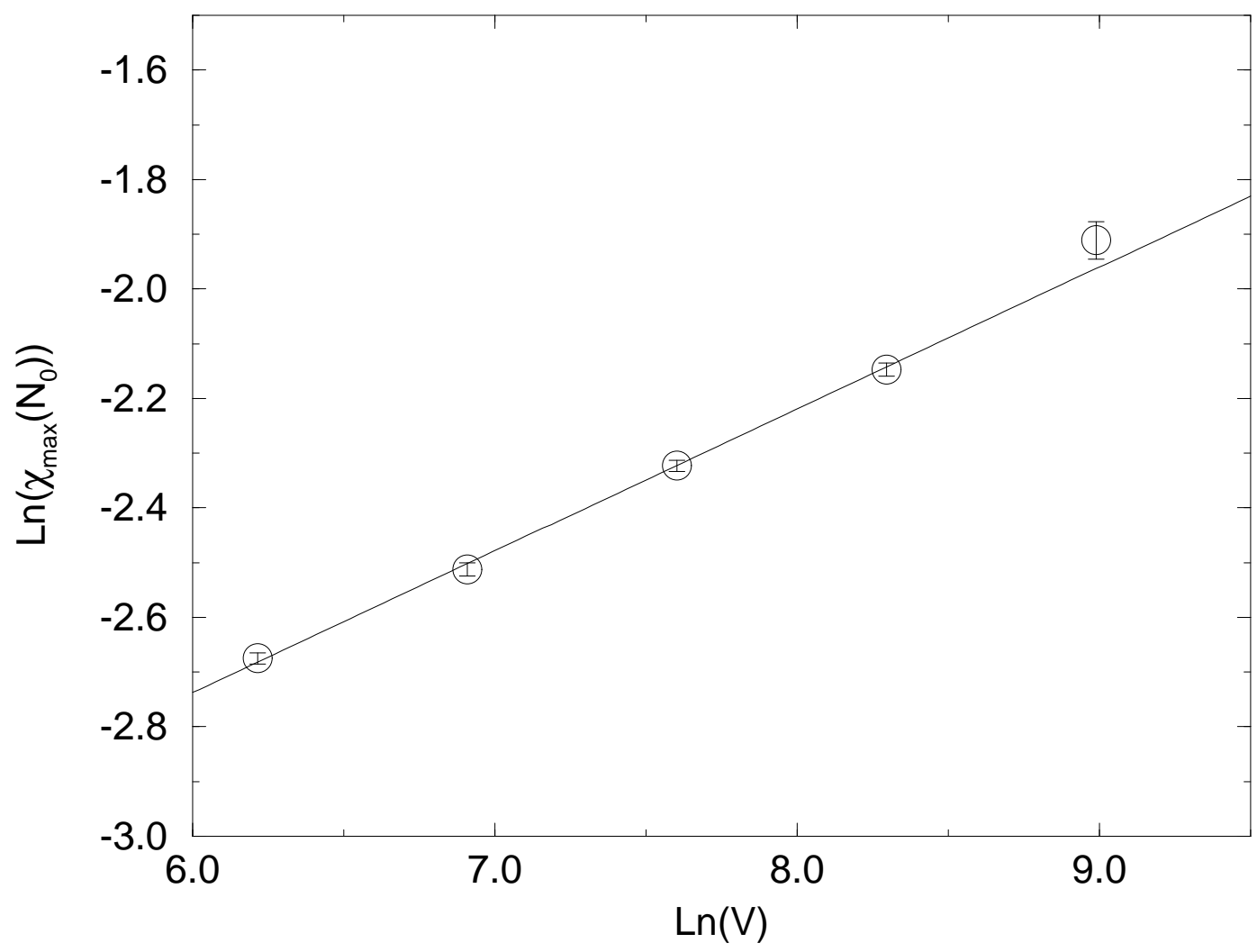

Figure 4: Log max node susceptibility 


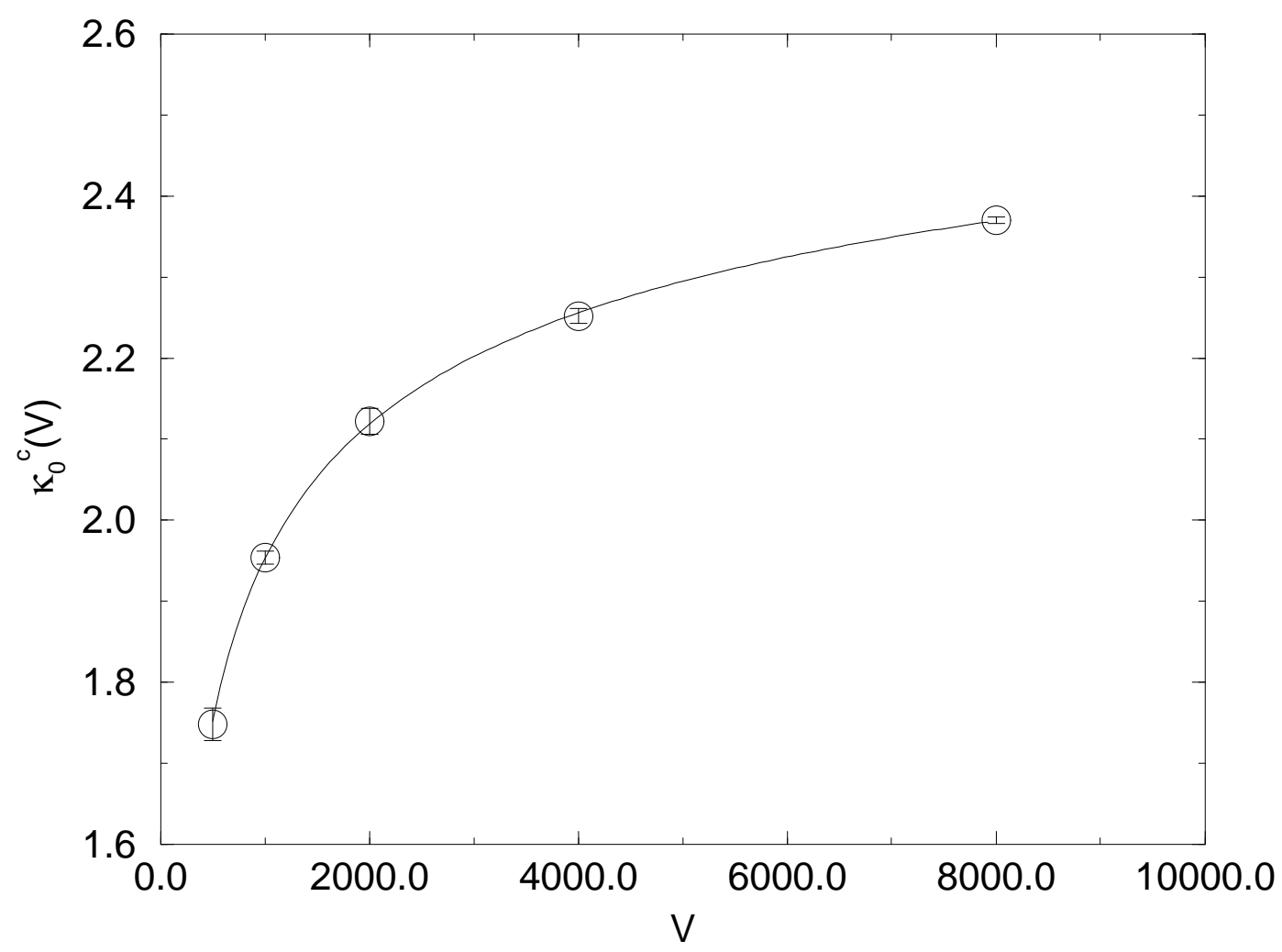

Figure 5: Critical coupling 